\title{
ZnO nanostructures prepared from ZnO:CNT mixtures
}

\author{
Y. H. Leung, ${ }^{1}$ A. B. Djurišić, ${ }^{1}$ M. H. Xie, ${ }^{1}$ C. Y. Kwong, ${ }^{2}$ W. K. Chan $^{3}$ \\ ${ }^{1}$ Department of Physics, The University of Hong Kong, Pokfulam Road, Hong Kong \\ ${ }^{2}$ Department of Electrical \& Electronic Engineering, The University of Hong Kong, Pokfulam \\ Road, Hong Kong \\ ${ }^{3}$ Department of Chemistry, The University of Hong Kong, Pokfulam Road, Hong Kong
}

\begin{abstract}
Due to its wide band gap $(3.37 \mathrm{eV})$ and large exciton binding energy $(60 \mathrm{meV}), \mathrm{ZnO}$ is of great interest for photonic applications. A number of different morphologies, such as nanobelts, nanowires, tetrapod nanostructures, tubular nanostructures, hierarchical nanostructures, nanobridges, nanonails, oriented nanorod arrays, nanoneedles, nanowalls, and nanosheets, were reported. A range of synthesis methods for fabrication of $\mathrm{ZnO}$ nanostructures was reported as well. A common method is evaporation from mixture of $\mathrm{ZnO}$ and carbon, which is usually in the form of graphite. In this work, we studied the morphology of the $\mathrm{ZnO}$ nanostructures fabricated from the mixture of $\mathrm{ZnO}$ (micron-sized and nanoparticles) and carbon (graphite, single-wall carbon nanotubes). When graphite and $\mathrm{ZnO}$ powders were used, tetrapod structures were obtained. If one of the reactants was nanosized, the diameter of the tetrapod arms was no longer constant. Finally, when both reactants were nanosized, novel morphologies were obtained. We studied the dependence of the morphology on the amount of starting material and the type of carbon used. The $\mathrm{ZnO}$ nanostructures were studied using field emission scanning electron microscopy, transmission electron microscopy, selected area electron diffraction, and X-ray diffraction. Growth mechanism and factors affecting the morphologies are discussed.
\end{abstract}

Keywords: $\mathrm{ZnO}$, nanostructures

\section{INTRODUCTION}

Synthesis of semiconductor nanostructures has attracted great attention due to their exceptional properties. Among all kinds of semiconductors, zinc oxide $(\mathrm{ZnO})$ has been recognized as a promising candidate for short wavelength photonic application due to its wide bandgap (3.37eV) and high exciton binding energy (60meV). The wide bandgap makes $\mathrm{ZnO}$ a suitable material for UV or blue emitting application, while the high exciton binding energy makes efficient exciton recombination possible even at room temperature. A number of nanostructures has been reported for $\mathrm{ZnO}$, such as nanowires, ${ }^{1-5}$ nanorods, ${ }^{6-9}$ tetrapod nanorods, ${ }^{5,9-13}$ nanobelt/ribbon structures, ${ }^{13-15}$ tubular nanostructures, ${ }^{16}$ and whisker nanostructures. ${ }^{17}$ Recently, novel morphologies like hierarchical structures, ${ }^{18}$ bridge/nailshaped nanostructures, ${ }^{19}$ multipod structures, ${ }^{10,20}$ nanoneedles, ${ }^{21}$ nanowalls, ${ }^{22}$ nanosheets, ${ }^{23}$ etc. have been demonstrated.

$\mathrm{ZnO}$ nanostructures have been fabricated by a range of synthesis methods, including the conventional thermal evaporation, ${ }^{1-3,5,8-24}$ metal organic vapor phase epitaxy (MOVPE), ${ }^{7,21}$ laser ablation, ${ }^{8}$ chemical synthesis, ${ }^{4,6}$ etc. Among all the methods, thermal evaporation/oxidation is the most commonly employed synthesis process. It is popular because of its relatively low-cost and easy to control. Pure $\mathrm{Zn}$, in pellet or powder form, has been used as an evaporation source. Dai et al. ${ }^{11}$ demonstrated synthesis of $\mathrm{ZnO}$ tetrapods by thermal oxidation of $\mathrm{Zn}$ powders in air at $925^{\circ} \mathrm{C}$. Similar works have also been reported by Roy et al. ${ }^{12}$ on the synthesis of $\mathrm{ZnO}$ tetrapods with nanowires grown from the end of the tetrapod nanorod by evaporation of $\mathrm{Zn}$ pellets in various atmospheres. Yan et al. ${ }^{13}$ also reported the fabrication of $\mathrm{ZnO}$ tetrapods with different shapes by evaporation of $\mathrm{Zn}$ powders in argon/oxygen mixture atmosphere at $800-900^{\circ} \mathrm{C}$. Other than pure $\mathrm{Zn}$, mixture of $\mathrm{ZnO}$ powders and carbon is also a well-known choice for a $\mathrm{Zn}$ source. $\mathrm{ZnO}$ is reduced to $\mathrm{Zn}$ or $\mathrm{Zn}_{1-\mathrm{x}} \mathrm{O}_{\mathrm{x}}$ by carbon at high temperatures. Graphite is commonly used as a carbon source although the use of active carbon and multiwalled carbon nanotubes ${ }^{24}$ has also been reported. Yao et al. ${ }^{2}$ reported mass production of different $\mathrm{ZnO}$ nanostructures, such as nanowires, nanoribbons, etc., by thermal evaporation of $\mathrm{ZnO}$ powders mixed with graphite. Banerjee et al. ${ }^{3}$ also reported synthesis of large quantity of $\mathrm{ZnO}$ nanowires by a similar process. Many $\mathrm{ZnO}$ novel 
nanostructures have also be synthesized by heating a $\mathrm{ZnO}$ :graphite mixture in the presence of catalysts. Hierarchical and bridge/nail-shaped $\mathrm{ZnO}$ nanostructures have been prepared by thermal evaporation of a powder mixture of $\mathrm{ZnO}$, indium oxide $\left(\mathrm{In}_{2} \mathrm{O}_{3}\right)$, and graphite. ${ }^{18-19}$ It is suggested that $\mathrm{In}_{2} \mathrm{O}_{3}$ nanostructures give extra surfaces for the growth of $\mathrm{ZnO}$, which eventually makes the exotic $\mathrm{ZnO}$ nanostructures. ${ }^{18}$ Park et al. ${ }^{23}$ demonstrated synthesis of $\mathrm{ZnO}$ nanosheets on gold $(\mathrm{Au})$ coated silicon substrates with width-to-thickness ratio up to one thousand by carbothermal reduction of $\mathrm{ZnO}$. Recently, we have also synthesized $\mathrm{ZnO}$ multipods by heating a mixture of $\mathrm{ZnO}$, germanium oxide $\left(\mathrm{GeO}_{2}\right)$, and graphite powder in a quartz tube at $1100^{\circ} \mathrm{C} .{ }^{10}$ It was observed that $\mathrm{ZnO}$ tetrapods instead of multipods were obtained without the presence of $\mathrm{GeO}_{2}$. Similar work was also demonstrated by Gao et al. ${ }^{20}$ on the synthesis of multipod-like $\mathrm{ZnO}$ whiskers with needle-like legs united at a common junction.

Possible applications of traditional nanostructures such as nanowires are straightforward, e.g. field emitters, junctions in electronics, etc. More complex structures like tetrapods also attracted considerable attention on their practical applications. $\mathrm{Xu}$ et al. $^{25}$ reported that $\mathrm{ZnO}$ tetrapods/acrylic resin composite had both anti-electrostatic and antibacterial functions. $\mathrm{ZnO}$ tetrapods have also been mixed with rubber to form a composite. ${ }^{26}$ The composite has improved tensile strength and wear resistance compared to natural rubber. ${ }^{26}$ A number of synthesis methods have been reported for $\mathrm{ZnO}$ tetrapod nanorod structures, from evaporation of pure $\mathrm{Zn}^{5,11-13}$ to carbothermal reduction of $\mathrm{ZnO}^{10}$ or $\mathrm{ZnCO}_{3}{ }^{9}$ The tetrapods demonstrated up to now have quite a variety of shapes, which seem to be affected by the fabrication processes. It was reported that the oxygen partial pressure had significant effect on the shape of the tetrapod. Yan et al. ${ }^{13}$ reported that tetrapods with uniform hexagonal arms were synthesized in relatively low oxygen pressure, while the arms' shape changed to trumpet-like when oxygen pressure was high. Roy et al. ${ }^{12}$ suggested that limited oxygen supply in the synthesis process could result in the growth of nanowires at the end of the tetrapods' arms.

In this work, $\mathrm{ZnO}$ nanostructures were fabricated from heating a mixture of $\mathrm{ZnO}$ (in the form of micro-sized powder or nano-particles) and carbon (in graphite form or single-walled carbon nanotubes (SWCNTs)). The influence of release rate of $\mathrm{Zn}$ vapor to the shape of the nanostructures was investigated. The vapor release rate was varied by the use of source materials with different sizes, and the amount of source materials. The obtained nanostructures were examined using scanning electron microscopy (SEM), transmission electron microscopy (TEM), selected area electron diffraction (SAED), and X-ray diffractometry (XRD). Growth mechanism and the factors affecting the morphologies were also discussed.

\section{EXPERIMENTAL DETAILS}

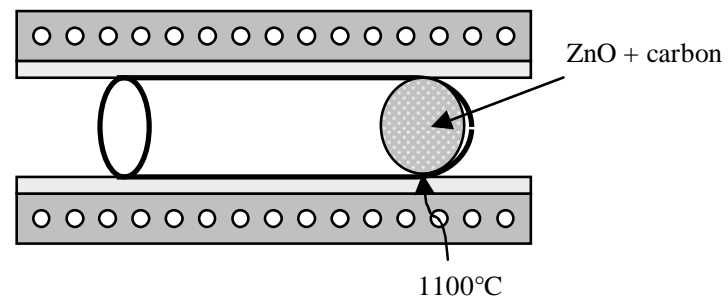

Figure 1. Illustration of the experimental setup.

The $\mathrm{ZnO}$ nanostructures were synthesized by thermally evaporating a mixed source of $\mathrm{ZnO}$ (micro-sized powder or nano-particles) and carbon (graphite or SWCNTs) at $1100^{\circ} \mathrm{C}$. Figure 1 shows a schematic diagram of the experimental setup. The $\mathrm{ZnO}$ powder (99.99\% purity) and nano-particles ( $7 \mathrm{~nm}$ crystallite size) were obtained from Aldrich and NanoScale Materials respectively. For the carbon source, the graphite ( $>99.5 \%$ purity) was obtained from International Laboratory while the SWCNTs (AP Grade) were acquired from Carbolex. The powders were mixed well ( $1: 1$ molar ratio of $\mathrm{ZnO}$ and carbon, $0.15 \mathrm{~g}$ of $\mathrm{ZnO}$ unless specified) and put in a quartz tube with one closed end. The quartz tube was then inserted into a horizontal tube furnace which was heated to the desired temperature. The open end of the quartz tube was exposed to the atmosphere. After 13 minutes of evaporation, white products were deposited on the inner wall of the open end of the quartz tube. The entire process took place at ambient atmosphere without catalyst. The obtained nanostructures were examined by scanning electron microscopy (SEM) using LEO 1530 FESEM, transmission electron microscopy (TEM) and selected area electron diffraction (SAED) using Philips Tecnai 20 TEM, and X-ray diffractometry (XRD) using Siemens D5000 X-ray diffractometer. 


\section{RESULTS AND DISCUSSIONS}

Figure 2 shows the representative SEM images of the $\mathrm{ZnO}$ nanostructures obtained from the four combinations of source materials. It can be observed that the nanostructures synthesized from heating a ZnO:graphite mixture exhibit tetrapod nanorod structures (Figure $2 \mathrm{a}$ and $2 \mathrm{~b}$ ). Such kind of structure is similar to our previous report from evaporation of pure $\mathrm{Zn}$ in air. ${ }^{12}$ Similar structure has also been reported by Dai et al. ${ }^{11}$ in which the arms extended from the core had a hexagonal cross-section and uniform diameter. When one of the starting materials becomes nano-sized, the morphologies of the nanostructures change significantly. Figure $2 \mathrm{c}$ and $2 \mathrm{~d}$ show the nanostructures from heating a ZnO:SWCNTs mixture. The arms of the tetrapods are no longer uniform in diameter. The diameter of the arms decreases gradually with the distance from the tetrapod core, and then increases to form a trumpet-like cap at the far end of the arms. The cross-section of the arms is not likely to be hexagonal, as observed from the images (Figure 2c and 2d). Similar morphologies were also obtained when $\mathrm{ZnO}$ nano-particles and graphite were used as the source materials (Figure 2e and 2f). Again, the diameter of the arms decreases with the distance away from the center core. However, arms with hexagonal cross-section were observed in some of the samples. Finally, when both source materials were nano-sized, novel morphologies were obtained. Figure $2 \mathrm{~g}$ and $2 \mathrm{~h}$ show the $\mathrm{ZnO}$ nanostructures obtained from heating a $\mathrm{ZnO}$ nano-particles:SWCNTs mixture. From the images, it is noticed that no tetrapods exist. Instead, straight nanorods with variable diameter are observed. There are mainly two kinds of nanorods observed from the sample: trumpet-like rods and bottle-like rods with thin "bottlenecks". It is also observed that some of the rods merge with one another (Figure 2h). Some of the structures look like tetrapods, but the angle between the arms is not fixed. This suggests that the formation of tetrapod-like structure is due to the random merging of separated nanorods instead of growth from a common core.

The influence of the amount of source materials on the resulting morphologies was also studied. Figure 3 shows the SEM images of the $\mathrm{ZnO}$ nanostructures synthesized from heating a ZnO nano-particles:SWCNTs mixture with different amount of source materials. Figure $3 \mathrm{a}$ and $3 \mathrm{~b}$ show the $\mathrm{ZnO}$ nanostructures obtained when $0.3 \mathrm{~g}$ of $\mathrm{ZnO}$ was used. These nanostructures exhibit nail-shaped structure which is similar to the nanonails previously demonstrated. ${ }^{19}$ In that report, nail-shaped nanostructures were synthesized from a mixture of $\mathrm{ZnO}$, indium oxide $\left(\mathrm{In}_{2} \mathrm{O}_{3}\right)$, and graphite. As no $\mathrm{In}_{2} \mathrm{O}_{3}$ was used in this work, we may conclude that the formation of those nail-shaped structures does not require any presence of $\operatorname{In}_{2} \mathrm{O}_{3}$ or indium in the source material. Instead, it is more likely to be dependent to the vapor pressure of $\mathrm{Zn}$ and oxygen. By comparing to the nanostructures obtained with the original amount of source material $(0.15 \mathrm{~g}$ of $\mathrm{ZnO})$, these nail-like structures exhibit slightly different morphologies. These rods have one broad end and one narrow end (Figure 3a and 3b) while the original structures have the smallest diameter in the middle and not the end of the rods (Figure $2 \mathrm{~g}$ and $2 \mathrm{~h}$ ). By further increasing the amount of source materials $(0.4 \mathrm{~g}$ or $0.6 \mathrm{~g}$ of $\mathrm{ZnO}$ ), it can be observed that the broad ends of the rods grow larger and exhibit hexagonal cross-sections (Figure 3c-f).

Figure 4 shows the XRD spectrum of the $\mathrm{ZnO}$ nanostructures obtained. The spectra in all cases show peaks corresponding to wurtzite $\mathrm{ZnO}$. All the obtained spectra show identical peaks, so that only one spectrum is shown for clarity. No diffraction peaks corresponding to $\mathrm{Zn}$ or other impurities were detected. TEM images of the $\mathrm{ZnO}$ nanostructures are shown on Figure 5. Some parts of the region show ripple-like contrast which is likely due to strain. The inset of Figure 5a shows the selected area electron diffraction (SAED) on one of the tetrapod arms. The SAED pattern indicates that the arms grow in (0001) direction. This agrees with our previous study. ${ }^{12}$ Similar findings were also reported on $\mathrm{ZnO}$ columns. ${ }^{27}$ From the images of the nail-shaped nanorods (Figure $2 \mathrm{c}-\mathrm{f}$ ), it can be observed that the morphology of the broad end of the rods looks similar to that of the $\mathrm{ZnO}$ hexagonal column in a recent study. ${ }^{27} \mathrm{We}$ can clearly notice the top face $(\{0001\}$ face, a.k.a. the $c$-face), side face $(\{10 \overline{10}\}$ face), and the $\{11 \overline{2} 3\}$ face inclining from the top face to the side face of the obtained nanostructures. It is very likely that the growth mechanism of the nanostructures reported here is similar to that of $\mathrm{ZnO}$ columns, which is related to the homoepitaxial nucleation of nanoscopic pyramids on the $\{0001\}$ face of the host. ${ }^{27}$ It was reported that this particular growth mechanism was attributed to the existence of an Ehrlich-Schwoebel barrier for diffusion of adspecies on the top $\{0001\} \mathrm{ZnO}$ surface. ${ }^{27}$ The mechanism behind the change of rod or arm diameter of the nanostructures is not fully understood and requires further studies. The SWCNTs employed in this work were used as received, without further purification. Therefore, it is also possible that the presence of metal impurities plays a role in the variation of morphologies. 

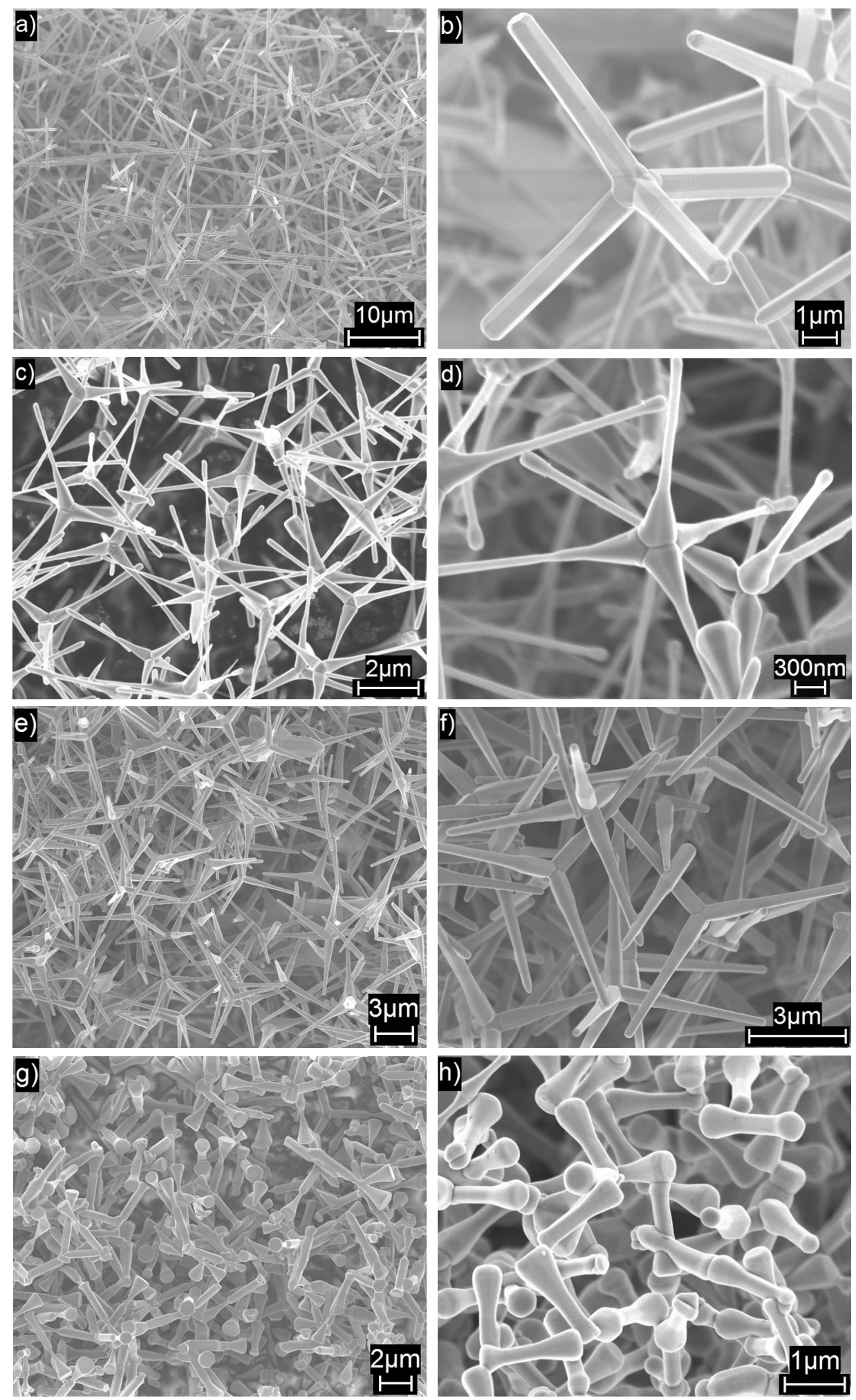

Figure 2. Representative SEM images of ZnO tetrapods fabricated by heating a mixture of a,b) ZnO:graphite, c,d) ZnO:SWCNTs, e,f) ZnO nano-particles:graphite, and g,h) ZnO nano-particles:SWCNTs. 

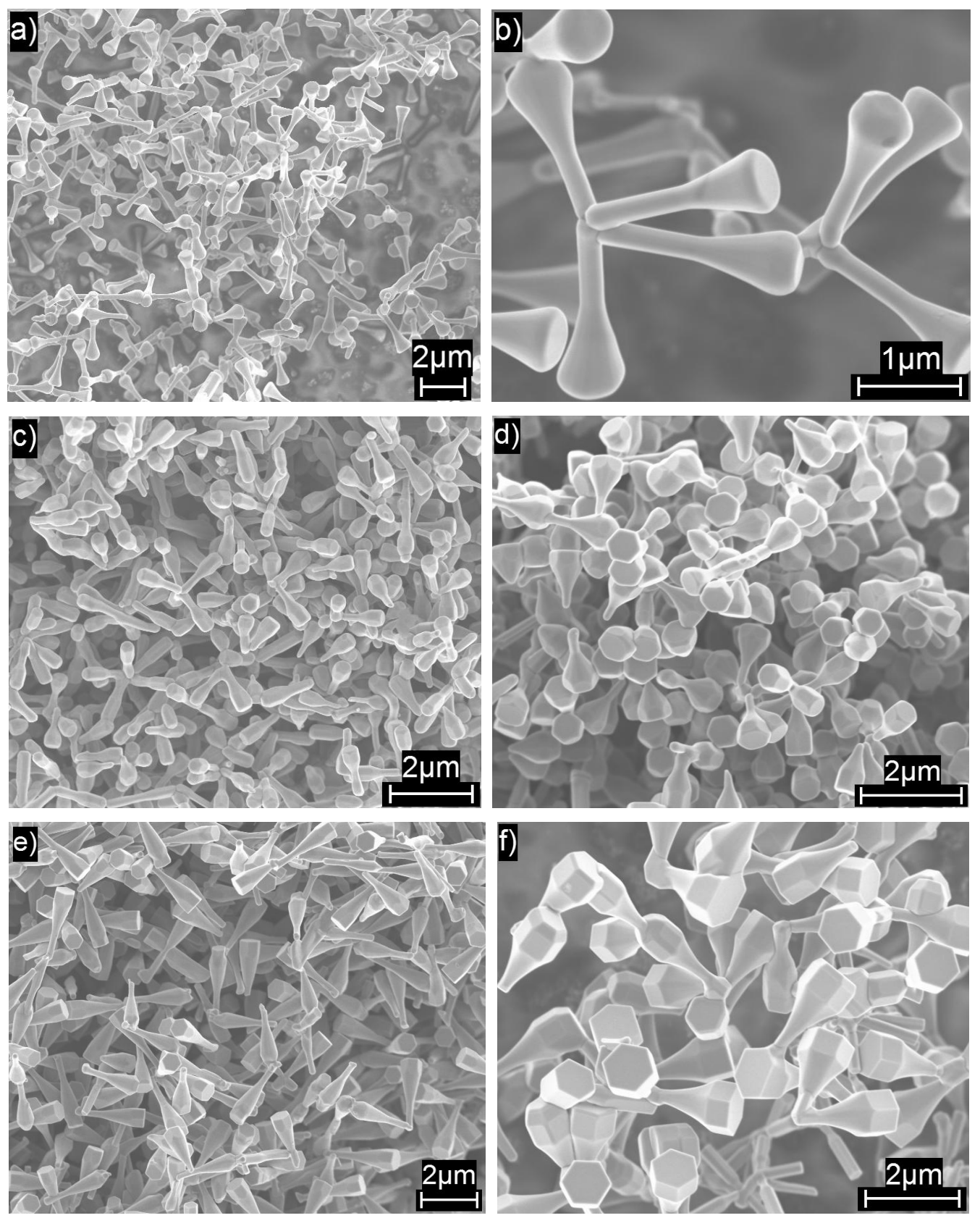

Figure 3. Representative SEM images of $\mathrm{ZnO}$ tetrapods fabricated by heating a mixture of $\mathrm{ZnO}$ nano-particles and SWCNTs with a,b) $0.3 \mathrm{~g}$, c,d) $0.45 \mathrm{~g}$, and e,f) $0.6 \mathrm{~g}$ of $\mathrm{ZnO}$. 


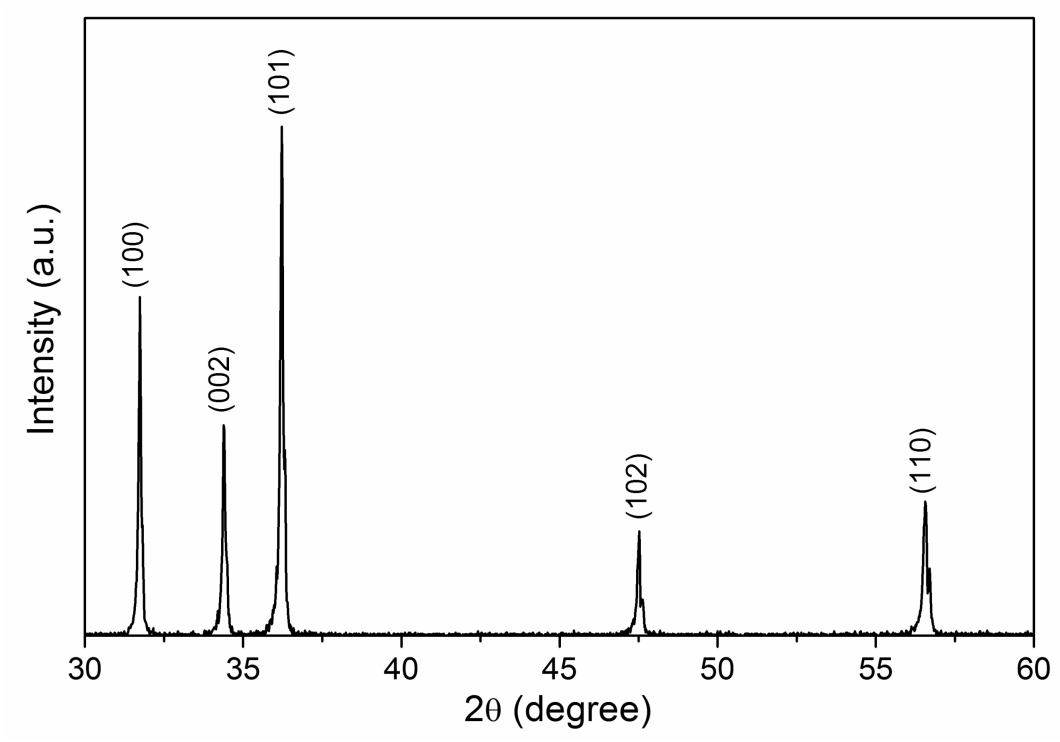

Figure 4. XRD spectrum of the $\mathrm{ZnO}$ nanostructures.
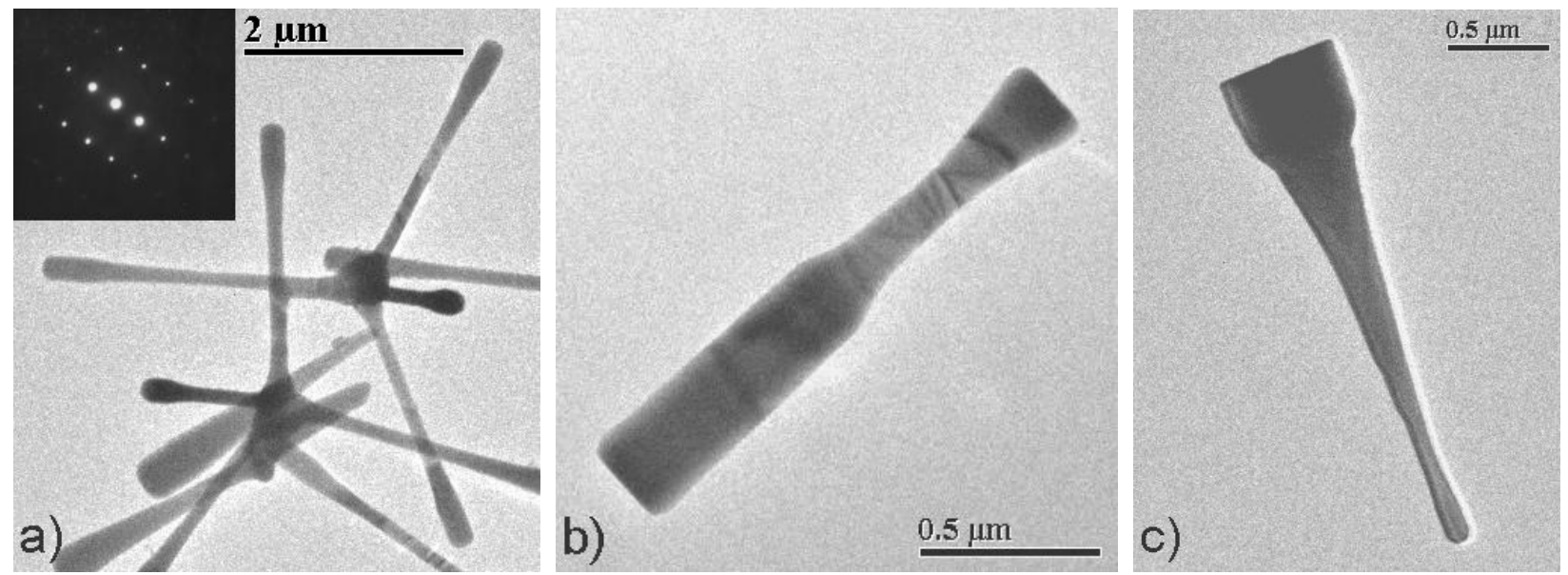

Figure 5. TEM images of a) $\mathrm{ZnO}$ tetrapods with variable arm diameter, b) bottle-shaped rod, and c) nail-shaped rod. Inset in a) shows the selected area electron diffraction (SAED) on one of the tetrapod arms.

\section{CONCLUSIONS}

In this work, we demonstrated a simple synthesis method to produce $\mathrm{ZnO}$ nanorods with different shapes and sizes by thermal evaporation of $\mathrm{ZnO}$ (micro-sized powder and nano-particles) and carbon (graphite and single-walled carbon nanotubes). When $\mathrm{ZnO}$ micro-sized powder and graphite were used, tetrapod structures were obtained. If one of the reactants was nanosized, the diameter of the tetrapod arms was no longer constant. Finally, when both reactants were nanosized, novel morphologies were obtained. We found that the variation in morphologies of the nanostructures was attributed to the change of $\mathrm{Zn}$ vapor release rate, resulted from the reactivity difference of the starting materials. However, the actual mechanism behind the morphology change is not fully understood and requires further studies. 


\section{ACKNOWLEGEMENTS}

The authors would like to thank Amy Wong and Wing Song Lee for FESEM examinations, and Dr. Ju Gao for XRD measurement. This work is supported by the University of Hong Kong University Research Committee seed funding grant. Financial support from the Research Grants Council of the Hong Kong Special Administrative Region, China is also acknowledged (HKU 7096/02P and 7009/03P).

\section{REFERENCES}

1. M. H. Huang, Y. Wu, H. Feick, N. Tran, E. Weber, and P. Yang, "Catalytic Growth of Zinc Oxide Nanowires by Vapor Transport", Adv. Mater. 13, 113-116 (2001).

2. B. D. Yao, Y. F. Chan, and N. Wang, "Formation of ZnO nanostructures by a simple way of thermal evaporation", Appl. Phys. Lett. 81, 757-759 (2002).

3. D. Banerjee, J. Y. Lao, D. Z. Wang, J. Y. Huang, Z. F. Ren, D. Steeves, B. Kimball, and M. Sennett, "Largequantity free-standing ZnO nanowires", Appl. Phys. Lett. 83, 2061-2063 (2003).

4. L. E. Greene, M. Law, J. Goldberger, F. Kim, J. C. Johnson, Y. Zhang, R. J. Saykally, and P. Yang, “LowTemperature Wafer-Scale Production of ZnO Nanowire Arrays", Augew. Chem. Int. Ed. 42, 3031-3034 (2003).

5. J. Park, H.-H. Choi, K. Siebein, and R. K. Singh, "Two-step evaporation process for formation of aligned zinc oxide nanowires", J. Crystal Growth 258, 342-348 (2003).

6. B. Liu, and H. C. Zeng, "Hydrothermal Synthesis of ZnO nanorods in the Diameter Regine of $50 \mathrm{~nm}$ ", J. Am. Chem. Soc. 125, 4430-4431 (2003).

7. W. I. Park, Y. H. Jun, S. W. Jung, and G.-C. Yi, "Excitonic emissions observed in ZnO single crystal nanorods", Appl. Phys. Lett. 82, 964-966 (2003).

8. A. B. Hartanto, X. Ning, Y. Nakata, and T. Okada, "Growth mechanism of ZnO nanorods from nanoparticles formed in a laser ablation plume", Appl. Phys. A 78, 299-301 (2003).

9. W. D. Yu, X. M. Li, and X. D. Gao, "Self-catalytic synthesis and photoluminescence of ZnO nanostructures on ZnO nanocrystal substrates", Appl. Phys. Lett. 84, 2658-2660 (2004).

10. A. B. Djurišić, Y. H. Leung, W. C. H. Choy, K. W. Cheah, and W. K. Chan, "Visible photoluminescence in ZnO tetrapod and multipod structures", Appl. Phys. Lett. 84, 2635-2637 (2004).

11. Y. Dai, Y. Zhang, Q. K. Li, and C. W. Nan, "Synthesis and optical properties of tetrapod-like zinc oxide nanorods", Chem. Phys. Lett. 358, 83-86 (2002).

12. V. A. L. Roy, A. B. Djurišić, W. K. Chan, J. Gao, H. F. Lui, and C. Surya, "Luminescent and structural properties of ZnO nanorods prepared under different conditions", Appl. Phys. Lett. 83, 141-143 (2003).

13. H. Yan, R. He, J. Pham, and P. Yang, "Morphogenesis of One-Dimensional ZnO Nano- and Microcrystals", Adv. Mater. 15, 402-405 (2003).

14. Z. W. Pan, Z. R. Dai, and Z. L. Wang, "Nanobelts of Semiconducting Oxides", Science 291, 1947-1949 (2001).

15. Y. B. Li, Y. Bando, T. Sato, and K. Kurashima, "ZnO nanobelts grown on Si substrate", Appl. Phys. Lett. 81, 144146 (2002).

16. Y. J. Xing, Z. H. Xi, X. D. Zhang, J. H. Song, R. M. Wang, J. Xu, Z. Q. Xue and D. P. Yu, “Nanotubular structures of zinc oxide", Solid State Comm. 129, 671-675 (2004).

17. Y. G. Wang, C. Yuen, S. P. Lau, S. F. Yu, and B. K. Tay, "Ultraviolet lasing of ZnO whiskers prepared by catalystfree thermal evaporation", Chem. Phys. Lett. 377, 329-332 (2003).

18. J. Y. Lao, J. G. Wen, and Z. F. Ren, "Hierarchical ZnO Nanostructures”, Nano Lett. 2, 1287-1291 (2002).

19. J. Y. Lao, J. Y. Huang, D. Z. Wang, and Z. F. Ren, "ZnO Nanobridges and Nanonails", Nano Lett. 3, 235-238 (2003).

20. T. Gao, Y. Huang, and T. Wang, "The synthesis and photoluminescence of multipod-like zinc oxide whiskers", J. Phys.: Condens. Matter 16, 1115-1121 (2004).

21. W.-I. Park, G.-C. Yi, M. Kim, and S. J. Pennycook, "ZnO Nanoneedles Grown Vertically on Si Substrates by NonCatalytic Vapor-Phase Epitaxy”, Adv. Mater. 14, 1841-1843 (2002).

22. J. Y. Lao, J. Y. Huang, D. Z. Wang, Z. F. Ren, D. Steeves, B. Kimball, and W. Porter, "ZnO nanowalls", Appl. Phys. A 78, 539-542 (2004).

23. J.-H. Park, H.-J. Choi, Y.-J. Choi, S.-H. Sohn, and J.-G. Park, “Ultrawide ZnO nanosheets", J. Mater. Chem. 14, 35-36 (2004). 
24. G. Gundiah, F. L. Deepak, A. Govindaraj, and C. N. R. Rao, "Carbothermal synthesis of the nanostructures of $\mathrm{Al}_{2} \mathrm{O}_{3}$ and $\mathrm{ZnO}$ ', Topics in Catalysis 24, 137-146 (2003).

25. T. Xu, and C. S. Xie, "Tetrapod-like nano-particle ZnO/acrylic resin composite and its multi-function property", Prog. Org. Coat. 46, 297-301 (2003).

26. Z. Zhou, S. Liu, and L. Gu, "Studies on the Strength and Wear Resistance of Tetrapod-Shaped ZnO WhiskerReinforced Rubber Composites", J. Appl. Polym. Sci. 80, 1520-1525 (2001).

27. J. B. Baxter, F. Wu, and E. S. Aydil, "Growth mechanism and characterization of zinc oxide hexagonal columns", Appl. Phys. Lett. 83, 3797-3799 (2003). 